\title{
Portable Multisensor Activity Monitor (SenseWear) Lacks Accuracy in Energy Expenditure Measurement during Treadmill Walking Following Stroke
}

\author{
Suzanne S Kuys ${ }^{1 *}$, Courtney Clark ${ }^{2}$ and Norman R Morris ${ }^{2}$ \\ ${ }^{1}$ Principal Research Fellow, Griffith Health Institute, Griffith University, Gold Coast, Australia; Metro North Hospital and Health Service, Queensland Health, Brisbane, \\ Australia \\ ${ }^{2}$ Griffith Health Institute, Griffith University, Gold Coast, Australia
}

\begin{abstract}
Introduction: This research aimed to assess the accuracy of a portable multi-sensor device (SenseWear armband) measuring energy expenditure in people with stroke compared with indirect calorimetry at rest and during treadmill walking. Secondary aims were to determine if there was a difference depending on which arm the device was placed and to determine the accuracy of the armband step count.
\end{abstract}

Materials and Methods: Ten stroke survivors (mean age 64.3 SD 7.7 years; $70 \%$ male) wore an armband on each arm and metabolic facemask. Energy expenditure was measured at rest and during two 10-minute bouts of treadmill walking at different speeds separated with a seated rest.

Results: The armband was accurate for measuring energy expenditure at rest (Intraclass correlations (ICC) $>0.869$ ), with poor to fair accuracy during treadmill walking (ICC $>0.306$ ). The non-hemiplegic arm provided more accurate energy expenditure measurement (ICC>0.409), underestimating with 10\%-15\% absolute percentage error. SenseWear armband was inaccurate for measuring step count (absolute percentage error approximately $30 \%$ ).

Conclusion: These results suggest that SenseWear armband lacks accuracy for measuring energy expenditure during treadmill walking in people with stroke; with the non-hemiplegic arm the most accurate. The armband is inaccurate measuring step count. Revision of algorithms specific for people following stroke may improve accuracy.

Keywords: Stroke; Energy expenditure; Physical activity; Treadmill

\section{Introduction}

Following stroke approximately two thirds of survivors have residual neurological deficits and impaired mobility which potentially promotes a sedentary lifestyle resulting in further deconditioning and functional decline [1]. An additional consequence following stroke further limiting mobility is the associated increased energy expenditure experienced with everyday tasks including walking [2]. It is possible that the mobility limitations and high levels of fatigue $[3,4]$ commonly experienced by stroke survivors is in part due to this high energy expenditure.

Accurate measurement of energy expenditure in stroke survivors in particular to complete everyday tasks has been difficult due to the lack of valid portable tools. Traditionally, the gold standard measures of energy expenditure are indirect calorimetry and doubly labelled water [5]. These measures however, are not suitable for everyday clinical settings; are expensive and invasive, have limited temporal resolution and require considerable expertise to conduct [5]; putting them beyond the scope of practising clinicians.

New innovative multisensory portable devices have been developed that are capable of measuring energy expenditure. One such device is the SenseWear Pro2 Armband (Bodymedia, Pittsburgh, PA, USA). This multi sensor device measures energy expenditure from five primary measurements: bi-axial accelerometers, galvanic skin response, skin temperature, near-body skin temperature and heat flux. These measurements are integrated using custom-written algorithms and estimates energy expenditure (in $\mathrm{kcal}$ and METS), step rate and sleep time. The SenseWear armband device has been compared with doubly labelled water assessment of energy expenditure during extended periods of free living in older adults [6] and people with stroke [7], with good accuracy. In older adults, the SenseWear armband has been shown to overestimate energy expenditure possibly due to changes in skin conductance and thermoregulation [8]. According to the manufacturer, the SenseWear armband is positioned over the triceps bulk of the upper right arm. There is some suggestion that the accuracy of the armband to measure energy expenditure might be reduced if normal arm swing does not occur; such as when walking slowly or using an elbow support frame [9]. In people with right sided hemiplegia reduced movement of the right arm also could influence the measurement accuracy. Therefore wearing these devices on the left arm needs to be validated in people with stroke. In addition these devices are able to determine step count, which has received little investigation.

The aim of this research was to determine the accuracy of a portable multisensory device (SenseWear armband) in measuring energy expenditure in people following stroke compared with indirect calorimetry via open circuit spirometric measurement of oxygen uptake at rest and during treadmill walking. Secondary aims were to determine

*Corresponding author: Suzanne Kuys, Griffith Health Institute, Griffith University, Gold Coast, The Prince Charles Hospital, Rode Rd, Chermside QLD 4032, Australia, Tel: 6173139 6349; Fax: 6173139 6228; Email: s.kuys@griffith.edu.au

Received February 28, 2014; Accepted March 25, 2014; Published April 30, 2014

Citation: Kuys SS, Clark C, Morris NR (2014) Portable Multisensor Activity Monitor (SenseWear) Lacks Accuracy in Energy Expenditure Measurement during Treadmill Walking Following Stroke. Int $\mathrm{J}$ Neurorehabilitation 1: 101. doi:10.4172/2376-0281.1000101

Copyright: $\odot 2014$ Kuys SS, et al. This is an open-access article distributed under the terms of the Creative Commons Attribution License, which permits unrestricted use, distribution, and reproduction in any medium, provided the original author and source are credited. 


\begin{tabular}{|l|l|l|}
\hline Characteristic & $\mathrm{N}(\%)$ & Mean (SD) \\
\hline Gender, male & $7(70)$ & $64.3(7.7)$ \\
\hline Age (years) & & $1.77(9.1)$ \\
\hline Height $(\mathrm{m})$ & & $88.7(17.6)$ \\
\hline Weight $(\mathrm{kg})$ & & $27(5)$ \\
\hline Body mass index $(\mathrm{kg} / \mathrm{m} 2)$ & $5(50)$ & \\
\hline Paretic side, right & & $78(51)$ \\
\hline Time post stroke $(\mathrm{months})$ & & $0.95(0.28)$ \\
\hline 10 m walk time $(\mathrm{m} / \mathrm{s})$ & & $394(103)$ \\
\hline Six minute walk test distance $(\mathrm{m})$ & & \\
\hline
\end{tabular}

Table 1: Participant characteristics

\begin{tabular}{|c|c|c|c|c|c|c|c|c|c|}
\hline $\begin{array}{l}\text { Participant } \\
\text { No. }\end{array}$ & Age & Gender & Height (cm) & Weight (kg) & $\begin{array}{l}\text { Hemiparetic } \\
\text { Side }\end{array}$ & $\begin{array}{l}\text { Time post stroke } \\
\text { (months) }\end{array}$ & $f$ & $\begin{array}{l}10 \mathrm{~m} \text { walk } \\
\text { time }(\mathrm{m} / \mathrm{s})\end{array}$ & $\begin{array}{l}\text { Six Minute Walk } \\
\text { (m) }\end{array}$ \\
\hline 1 & 50 & Male & 170 & 65 & Right & 2 & Nil & 1.25 & 438 \\
\hline 2 & 70 & Male & 190 & 110 & Left & 86 & Nil & 0.98 & 375 \\
\hline 3 & 75 & Male & 184 & 94 & Right & 17 & Nil & 1.48 & 572 \\
\hline 4 & 64 & Male & 183 & 80 & Right & 101 & Single stick & 0.63 & 228 \\
\hline 5 & 71 & Female & 168 & 72 & Left & 100 & Single stick & 0.53 & 494 \\
\hline 6 & 69 & Male & 181 & 120 & Right & 187 & Single stick & 0.75 & 247 \\
\hline 7 & 61 & Female & 157 & 70 & Left & 24 & Nil & 1.26 & 446 \\
\hline 8 & 69 & Male & 181 & 106 & Left & 75 & Nil & 1.11 & 402 \\
\hline 9 & 53 & Male & 190 & 81 & Right & 101 & Nil & 0.96 & 301 \\
\hline 10 & 61 & Female & 169 & 89 & Left & 85 & Single stick & 0.67 & 394 \\
\hline
\end{tabular}

Table 2: Individual participant demographics

\begin{tabular}{|c|c|c|c|c|c|c|c|c|c|}
\hline \multirow[b]{2}{*}{ Measure } & \multirow{2}{*}{$\begin{array}{l}\text { Indirect Calorimetry/ } \\
\text { Observations }\end{array}$} & \multirow{2}{*}{$\begin{array}{l}\text { Non Hemi arm } \\
\text { armband }\end{array}$} & \multirow{2}{*}{$\begin{array}{l}\text { Hemi arm } \\
\text { armband }\end{array}$} & \multicolumn{2}{|l|}{ Mean Difference } & \multicolumn{2}{|c|}{ Absolute $\%$ error } & \multicolumn{2}{|l|}{ ICC } \\
\hline & & & & IC minus Non Hemi & IC minus Hemi & $\begin{array}{l}\text { IC vs Non } \\
\text { Hemi }\end{array}$ & IC vs Hemi & $\begin{array}{l}\text { IC vs Non } \\
\text { Hemi }\end{array}$ & IC vs Hemi \\
\hline \multicolumn{10}{|c|}{ Energy expenditure (kcal/min) } \\
\hline At rest & $1.43(0.26)$ & $1.31(0.26)$ & $1.15(0.25)$ & 0.22 (0.08 to 0.37$)$ & $\begin{array}{l}0.28 \text { ( } 0.16 \text { to } \\
0.42)\end{array}$ & $16.1 \%$ & $21.7 \%$ & 0.869 & 0.886 \\
\hline $\begin{array}{l}\text { Comfortable } \\
\text { pacev }\end{array}$ & $4.45(1.5)$ & $4.37(2.12)$ & $5.29(0.95)$ & $\begin{array}{l}0.3441(-1.36 \text { to } \\
2.18)\end{array}$ & $\begin{array}{l}-0.85(-2.10 \text { to } \\
0.41)\end{array}$ & $9.3 \%$ & $17.5 \%$ & 0.650 & 0.306 \\
\hline Fast pace & 5. $05(1.7)$ & $5.02(2.18)$ & $5.66(1.17)$ & $0.57(-2.43$ to 3.58$)$ & $\begin{array}{l}-0.19(-3.55 \text { to } \\
3.45)\end{array}$ & $11.3 \%$ & $36.4 \%$ & 0.409 & 0.404 \\
\hline \multicolumn{4}{|c|}{ Number of steps (n) } & $\begin{array}{l}\text { Observation minus } \\
\text { Non Hemi }\end{array}$ & $\begin{array}{l}\text { Observation } \\
\text { minus Hemi }\end{array}$ & & & & \\
\hline $\begin{array}{l}\text { Comfortable } \\
\text { pace }\end{array}$ & $93(17)$ & $60(42)$ & $68(33)$ & 25 (-24 to 87$)$ & $\begin{array}{l}29 \\
(6 \text { to } 52)\end{array}$ & $32.7 \%$ & $36.0 \%$ & 0.601 & 0.780 \\
\hline Fast pace & $107(18)$ & $66(39)$ & $87(33)$ & $\begin{array}{l}31 \\
\text { to } 87)\end{array}$ & $\begin{array}{l}16 \\
(-9 \text { to } 41)\end{array}$ & $35.8 \%$ & $16.5 \%$ & 0.841 & 0.905 \\
\hline
\end{tabular}

ICC: Intraclass Correleation Coefficient; IC: Indiect Calorimetry

Table 3: Mean (SD) energy expenditure and step count at rest, during comfortable and fast treadmill pace

if there was a difference in which arm was used for the placement of the armband device and the accuracy of the SenseWear armband step count. We hypothesised that the SenseWear armband would be accurate for measuring energy expenditure in stroke survivors at rest and during two externally paced treadmill walking speeds. Additionally we hypothesized that the non-hemiplegic arm would be more accurate for measuring energy expenditure.

\section{Materials and Methods}

\section{Participants, therapists, centres}

Participants were recruited from Stroke Support Groups within the Brisbane and Gold Coast area to participate in this study. To be eligible participants were required to have a diagnosis of stroke, be aged between 18 and 80 years, and able to walk independently with or without an aid at the speed of at least $0.3 \mathrm{~m} / \mathrm{s}$. Individuals were excluded if they had co-morbidities relating to the lower limbs that could limit their gait such as arthritis or Parkinson disease; had an unstable cardiac status which could preclude them from participating in submaximal exercise sessions; or if they were unable to understand the instructions and provide informed consent. Institutional Human Research ethics committee provided approval for this study.

\section{Devices}

Energy expenditure was measured continuously using indirect calorimetry at rest and during exercise. The metabolic cart/calorimeter (Cosmed, Quark, CPET, Rome, Italy) was calibrated for volume, using a 3L syringe (Hans-Rudolph, Kansas City, MO, USA) and the expired fraction of oxygen and carbon dioxide using alpha grade calibration gases (BOC gases, Labrador, QLD, Australia). During the test participants were fitted with a face-mask for the collection of expired gases to allow for the estimation of oxygen consumption. At the 
completion of the rest and exercise periods, data from the metabolic cart was downloaded and averaged over 1-minute periods.

The SenseWear armband device was worn on each arm, positioned over the triceps bulk, according to manufacturer specifications. To determine if there was a difference between the arms to which the device is fitted, one armband was fitted to the hemiplegic arm and another to the non-hemiplegic arm. The armband was fitted at least 10 minutes prior to the commencement of the measurement of resting energy expenditure commenced (see procedures section). At the completion of the rest and exercise periods, data from the armband was downloaded in 1-minute intervals. Data from the armband was then time-aligned with the data from the metabolic cart.

\section{Procedures}

Participants attended one two-hour testing session at Griffith University, and were instructed to refrain from performing any strenuous exercise or consuming coffee on the day of testing to ensure that energy expenditure would not be elevated [10]. Demographic data was recorded upon arrival, including weight, height, date of birth, date of stroke, side of hemiplegia, walking aids, other co-morbidities and current medications. Walking ability was measured using a 10 metre walk test with a moving start walking at a self-selected pace [11] and a 6 min walk test [12]. Participants were permitted to rest as required.

Following familarisation participants were fitted with the armband devices and the facemask for indirect calorimetry measurements. Participants then lay supine for $15 \mathrm{~min}$ to measure energy expenditure at rest. Each participant then completed two 10-minute bouts of treadmill walking at different speeds with a seated rest in between. During each exercise bout participants walked on a treadmill for 10 mins, allowing warm up for several minutes until steady state heart rate was achieved. For the first bout, participants walked at a self-determined comfortable pace, were able to stop at any time and were encouraged not to hold on the handrail throughout the treadmill walking. After a 5 min seated rest participants again walked on the treadmill for 10 mins, this time at a faster pace. All participants had prior experience walking on a treadmill following their stroke. No bodyweight support was provided during treadmill bouts.

\section{Outcome measures}

Accuracy of the SenseWear armband device was determined by comparing energy expenditure $(\mathrm{kcal} / \mathrm{min})$ recorded by each armband, hemiplegic and non-hemiplegic arm, with the indirect calorimetry data from the metabolic cart. Data was compared at rest and during treadmill walking. In addition the number of steps measured by the armbands during each 10-minute bout was compared with a manual step count. The manual step count was determined using a hand counter in whereby an independent observer counted each step individually.

\section{Data analysis}

Descriptive statistics were used to quantify energy expenditure at rest and during each treadmill walking bout during the last five minutes to enable steady state to be reached for each condition. Descriptive statistics described the number of steps throughout each treadmill walking bout. Mean (95\% confidence interval) differences between the SenseWear armband and indirect calorimetry for measurement of energy expenditure and manual count for step count were determined using paired t-tests. Accuracy of the SenseWear armband to measure energy expenditure and number of steps was determined using Intraclass correlations (ICC2,1) using SPSS v 19.0. An ICC value of $>$
0.75 was interpreted to be good and $>0.9$ was deemed excellent [13]. Absolute percentage error (APE) was calculated by using the formula: |mean difference / average of the two measurements x 100. The significance level of all analyses was set to $\mathrm{p}<0.05$.

\section{Results \\ Participants}

Ten stroke survivors participated in this study, 7 men and 3 women. Participants had an average age of 64.3 (SD 7.7) years and were 79 (SD 57) months post stroke (Tables 1 and 2). Fifty percent had their dominant side affected by the stroke. Four participants did not complete the fast paced treadmill walk bout due to fatigue and safety issues. The average (SD) treadmill belt speed for the comfortable paced bout was $0.8 \mathrm{~m} / \mathrm{s}(0.2)$ and for fast paced bout, $1.1 \mathrm{~m} / \mathrm{s}(0.3)$.

\section{Energy expenditure}

Table 3 presents mean (SD) energy expenditure at rest and during each treadmill bout measured with indirect calorimetry and each armband. Mean (95\% confidence interval, CI) differences, absolute percentage error and intraclass correlation analyses between the hemiplegic and non-hemiplegic armband with indirect calorimetry are also presented. Resting energy expenditure determined using indirect calorimetry was 1.43 (SD 0.26$) \mathrm{kcal} / \mathrm{min}$. This was higher than that measured using the SenseWear armband. The Sensewear armband underestimated resting energy expenditure, regardless of which arm it was measured on.

During treadmill walking, energy expenditure measured on the non-hemiplegic arm was underestimated compared to indirect calorimetry; mean difference comfortable pace $0.34 \mathrm{kcal} / \mathrm{min}(95 \% \mathrm{CI}$ -1.36 to 2.18 ) and fast pace $0.57 \mathrm{kcal} / \mathrm{min}$ ( $95 \% \mathrm{CI}-2.43$ to 3.58 ). Energy expenditure measures on the hemiplegic arm were overestimated during both comfortable pace treadmill walking (mean difference $-0.85 \mathrm{kcal} / \mathrm{min} 95 \% \mathrm{CI}-2.10$ to 0.14$)$ and fast pace treadmill walking (mean difference $-0.19 \mathrm{kcal} / \mathrm{min} 95 \%$ CI -3.55 to 3.45 ). SenseWear armband had good accuracy for measuring energy expenditure at rest for both arms (ICC >0.869). Accuracy was lower during treadmill walking. Energy expenditure during treadmill walking measured on the non-hemiplegic arm had fair to moderate accuracy (ICC >0.409) while accuracy was low for the hemiplegic arm (ICC >0.306). Absolute percentage error ranged from $9.3 \%$ for comfortable paced treadmill walking to $16.1 \%$ at rest for the non-hemiplegic arm. For the hemiplegic arm, absolute percentage error was unacceptably high, $>17.5 \%$.

\section{Step count}

Table 3 presents mean (SD) step count during each treadmill walking bout measured by direct observation and with each armband. Mean differences (95\% confidence interval), absolute error and intraclass correlation analyses between the hemiplegic and nonhemiplegic armband are also presented. Step count measures recorded on both the hemiplegic and non-hemiplegic armbands undercounted compared to direct observations. Intraclass correlations indicate moderate to good validity for step count during both comfortable and fast paced treadmill walking for armbands worn on both the hemiplegic and non-hemiplegic arm. However, absolute percentage errors are high; ranging from $16 \%$ to $36 \%$.

\section{Discussion}

The aim of this study was to investigate the validity of the SenseWear armband device for measuring energy expenditure and steps during a 
graded treadmill exercise test. The SenseWear armband demonstrated good accuracy for measuring energy expenditure at rest for both the hemiplegic and non-hemiplegic arm. Accuracy during treadmill walking, regardless of speed, was moderate with the non-hemiplegic arm and fair with the hemiplegic arm. It appears that during treadmill walking, the SenseWear armband is more accurate when placed on the non-hemiplegic arm. Step count was not accurately measured in this group of stroke survivors.

Previous studies have shown conflicting results regarding the accuracy of the SenseWear armband in stroke survivors. Moore et al. [7] found good agreement between the armband and doubly labeled water for measuring free-living energy expenditure; with the armband underestimating energy expenditure by approximately $4 \%$. However, another recent study [14] found that the armband overestimated energy expenditure compared to a metabolic cart. Perhaps one reason for these differences is the comparative measure as well as the arm being tested. SenseWear armband manufacturers recommend that the armband be worn on the right arm. In people with hemiplegia this may not always reflect the swinging arm. Although it would be anticipated that better accuracy would be found on the functional swinging arm; this may also depend on the level of walking limitation. In people with mild gait deficits, who don't require the use of an aid, applying the armband onto the non-hemiplegic arm may result in better accuracy. For those stroke survivors who require a gait aid such as a single or four-point stick, arm swing is not necessarily natural and may influence measures. Certainly this is one possible explanation for finding better accuracy for energy expenditure measured on the non-hemiplegic arm [1]. Interestingly, Manns [1] found that the SenseWear armband overestimated energy expenditure regardless of which arm was measured. We found that energy expenditure during treadmill walking was underestimated when measured on the non-hemiplegic arm but was overestimated on the hemiplegic arm. However, for the current study, the mode of walking test is likely to have influenced our results. Energy expenditure was required to be measured by a non-portable metabolic cart, requiring a treadmill walk test to be used. Walking on a treadmill has some differences to over ground walking [15] requiring potentially greater balance and attention demands [16]. Although, stroke survivors have demonstrated no detrimental effects on walking pattern while walking on a treadmill [17], we found in this study that the majority of participants used the handrail on their non-hemiplegic side for safety and confidence often leaving their hemiplegic arm to swing. It is also possible that different levels of accuracy may have been found if over ground walking was used.

The SenseWear armband was not accurate for measuring step count. Absolute percentage error for step count was approximately $30 \%$ for both arms at both treadmill walk speeds. This finding supports a recent study which also highlighted poor accuracy of the armband for measuring step count in a group of mild to moderately affected stroke survivors during an over ground walk test [14]. Gait speed may be a factor in the accuracy of the SenseWear armband to determine step count. Investigations with other populations have found under counting at slower walking speeds $[9,18]$ but this requires further investigation. Despite, the high error for step count, good correlations with ICCs $>0.6$ ) were demonstrated, regardless of treadmill walking pace and the arm wearing the SenseWear armband. This is difficult to explain. Perhaps, it is possible that the armband may be clinically useful to indicate step count in people with stroke as long as clinicians are aware of the $30 \%$ error rate.

This study has some limitations. The sample size is small and there is a range of walking ability of participants recruited to this study limiting the generalizability of the findings. In addition, we planned to have all participants complete two walking tests, a comfortable and fast pace test to examine the ability of the armband to detect differences in energy expenditure across these two speeds. Due to the heterogeneity of our participants we were not able to specify the treadmill speed for all participants. Four participants were deemed by study investigators as unsafe to complete the second treadmill walk; limiting our findings for the fast treadmill test. The current study's findings are preliminary and caution is needed when generalizing these findings. Further studies are required with larger samples encompassing the range of walking disability observed in this population.

\section{Conclusion}

In conclusion, our results suggest that the SenseWear armband is accurate for measuring energy expenditure at rest. However, some caution is advised, for slow walkers following stroke during treadmill walking. Best accuracy is obtained when the device is worn on the nonhemiplegic arm; with energy expenditure underestimated by between $10 \%$ and $15 \%$. The SenseWear armband is not accurate for measuring step count.

\section{Ethics Approval}

Griffith University Human Research Ethics Committee approved this study. All participants gave written informed consent before data collection began.

\section{Acknowledgements}

The authors would like to acknowledge, C Louwen, T Richards, A Miller, B Kvamasa for their assistance with data collection and interpretation

\section{References}

1. Gebruers N, Vanroy C, Truijen S, Engelborghs S, De Deyn PP (2010) Monitoring of physical activity after stroke: a systematic review of accelerometry-based measures. Arch Phys Med Rehabil 91: 288-297.

2. Platts MM, Rafferty D, Paul L (2006) Metabolic cost of over ground gait in younger stroke patients and healthy controls. Med Sci Sports Exerc 38: 1041 1046

3. Lewis SJ, Barugh AJ, Greig CA Saunders DH, Fitzsimons C et al. (2011) Is fatigue after stroke associated with physical deconditioning? A cross-sectional study in ambulatory stroke survivors. Arch Phys Med Rehabil 92: 295-298.

4. Mead GE, Graham C, Dorman P, Bruins SK, Lewis SC, et al. (2011) Fatigue after stroke: baseline predictors and influence on survival. Analysis of data from UK patients recruited in the International Stroke Trial. PLoS One 6: e16988.

5. Casaburi R (2007) Activity monitoring in assessing activities of daily living COPD 4: 251-255

6. Mackey DC, Manini TM, Schoeller DA, Koster A, Glynn NW, et al. (2011) Validation of an armband to measure daily energy expenditure in older adults. J Gerontol A Biol Sci Med Sci 66: 1108-1113.

7. Moore SA, Hallsworth K, Bluck LJ, Ford GA, Rochester L, et al. (2012) Measuring energy expenditure after stroke: validation of a portable device. Stroke 43: 1660-1662.

8. Heiermann S, Khalaj Hedayati K, Müller MJ, Dittmar M (2011) Accuracy of a portable multisensor body monitor for predicting resting energy expenditure in older people: a comparison with indirect calorimetry. Gerontology 57: 473-479.

9. Hill K, Dolmage TE, Woon L, Goldstein R, Brooks D (2010) Measurement properties of the SenseWear armband in adults with chronic obstructive pulmonary disease. Thorax 65: 486-491.

10. Hursel R, Viechtbauer W, Dulloo AG, Tremblay A, Tappy L, et al. (2011) The effects of catechin rich teas and caffeine on energy expenditure and fat oxidation: a meta-analysis. Obes Rev 12: e573-581.

11. Wade DT, Wood VA, Heller A, Maggs J, Langton Hewer R (1987) Walking after stroke. Measurement and recovery over the first 3 months. Scand J Rehabil Med 19: 25-30. 
Citation: Kuys SS, Clark C, Morris NR (2014) Portable Multisensor Activity Monitor (SenseWear) Lacks Accuracy in Energy Expenditure Measurement during Treadmill Walking Following Stroke. Int J Neurorehabilitation 1: 101. doi:10.4172/2376-0281.1000101

12. Enright PL (2003) The six-minute walk test. Respir Care 48: 783-785.

13. Portney LG, Watkins MP (2008) Foundations of Clinical Research: Applications to Practice (3rd Ed) 2008: Upper Saddle River, NJ : Prentice Hall.

14. Manns PJ, Haennel RG (2012) SenseWear Armband and Stroke: Validity of Energy Expenditure and Step Count Measurement during Walking. Stroke Res Treat 2012: 247165.

15. Wass E, Taylor NF, Matsas A (2005) Familiarisation to treadmill walking in unimpaired older people. Gait Posture 21: 72-79.
16. Bayat R, Barbeau H, Lamontagne A (2005) Speed and temporal-distance adaptations during treadmill and overground walking following stroke. Neurorehabil Neural Repair 19: 115-124.

17. Kuys SS, Brauer SG, Ada L, Russell TG (2008) Immediate effect of treadmill walking practice versus overground walking practice on overground walking pattern in ambulatory stroke patients: an experimental study. Clin Rehabil 22 931-939.

18. Dwyer TJ, Alison JA, McKeough ZJ, Elkins MR, Bye PT (2009) Evaluation of the SenseWear activity monitor during exercise in cystic fibrosis and in health Respir Med 103: 1511-1517. 